\title{
Article
}

\section{Zero Average Surface Controlled Boost-Flyback Converter}

\author{
Juan-Guillermo Muñoz ${ }^{1, *} \mathbb{( D}$, Fabiola Angulo ${ }^{2}$ and David Angulo-Garcia ${ }^{3}(\mathbb{D}$ \\ 1 Instituto Tecnológico Metropolitano, Calle 54A \# 30-01, Barrio Boston, Medellín 050013, Colombia \\ 2 Departamento de Ingeniería Eléctrica, Electrónica y Computación-Bloque Q, Facultad de Ingeniería y \\ Arquitectura, Campus La Nubia, Universidad Nacional de Colombia-Sede Manizales, \\ Manizales 170003, Colombia; fangulog@unal.edu.co \\ 3 Grupo de Modelado Computacional-Dinámica y Complejidad de Sistemas, Instituto de Matemáticas \\ Aplicadas, Universidad de Cartagena, Carrera 6 \# 36-100, Cartagena de Indias 130001, Colombia; \\ dangulog@unicartagena.edu.co \\ * Correspondence: juanmunozc@itm.edu.co
}

Citation: Muñoz, J.-G.; Angulo, F.; Angulo-García, D. Zero Average Surface Controlled Boost-Flyback Converter. Energies 2021, 14, 57. https://doi.org/10.3390/ en14010057

Received: 14 November 2020 Accepted: 18 December 2020 Published: 24 December 2020

Publisher's Note: MDPI stays neutral with regard to jurisdictional clai$\mathrm{ms}$ in published maps and institutional affiliations.

Copyright: (C) 2020 by the authors. Licensee MDPI, Basel, Switzerland. This article is an open access article distributed under the terms and conditions of the Creative Commons Attribution (CC BY) license (https:// creativecommons.org/licenses/by/ $4.0 /)$.

\begin{abstract}
The boost-flyback converter is a DC-DC step-up power converter with a wide range of technological applications. In this paper, we analyze the boost-flyback dynamics when controlled via a modified Zero-Average-Dynamics control technique, hereby named Zero-Average-Surface (ZAS). While using the ZAS strategy, it is possible to calculate the duty cycle at each PWM cycle that guarantees a desired stable period-1 solution, by forcing the system to evolve in such way that a function that is constructed with strategical combination of the states over the PWM period has a zero average. We show, by means of bifurcation diagrams, that the period- 1 orbit coexists with a stable period-2 orbit with a saturated duty cycle. While using linear stability analysis, we demonstrate that the period- 1 orbit is stable over a wide range of parameters and it loses stability at high gains and low loads via a period doubling bifurcation. Finally, we show that, under the right choice of parameters, the period-1 orbit controller with ZAS strategy satisfactorily rejects a wide range of disturbances.
\end{abstract}

Keywords: boost-flyback converter; Zero Average Dynamics control; Zero Average Surface control; bifurcation diagram; Floquet multipliers

\section{Introduction}

Power converters are electronic circuits whose aim is to adjust the output voltage to a desired fixed value (regulation task) or to a defined time-dependent function (tracking task). Power converters can be classified into step-down or step-up, depending on the output/input voltage ratio. The former (latter) takes an input voltage and outputs a lower (higher) value than the input. The boost converter is one the most prominent examples of a step-up architecture, mostly due to its simple design and high efficiency for unelevated values of the duty cycle [1]. However, as the output/input ratio (gain) increases in the boost converter, its efficiency drops. This issue highlights the challenges in step-up converters in order to find highly-efficient designs with high gain capabilities. Solutions to this problem have been previously proposed [2-5], while using complex designs. These new designs involve an increased number of diodes, transistors, capacitors, and coils, which renders the analysis of these systems impractical. In many cases, the only techniques that are available for analyzing these systems are numerical simulations and averaged models. Unfortunately, those techniques can neither determine the behavior of the system in a wide operation range nor consider the effects of nonlinear phenomena.

The use of magnetically coupled inductors is one of the main ideas exploited so far in the design of highly efficient step-up converters (see [6] for a list of applications). The boostflyback converter that was proposed by [7-9] is probably the simplest converter with high gain and high efficiency. It integrates two different converter topologies: boost and flyback, which operate with two magnetically coupled coils (see Figure 1 for illustration). Despite the advantages of the boost-flyback converter, it was only analyzed in deep for the 
first time in [10], while using an average model. Thereafter, the research devoted to the analysis and control of this power converter has increased [11].

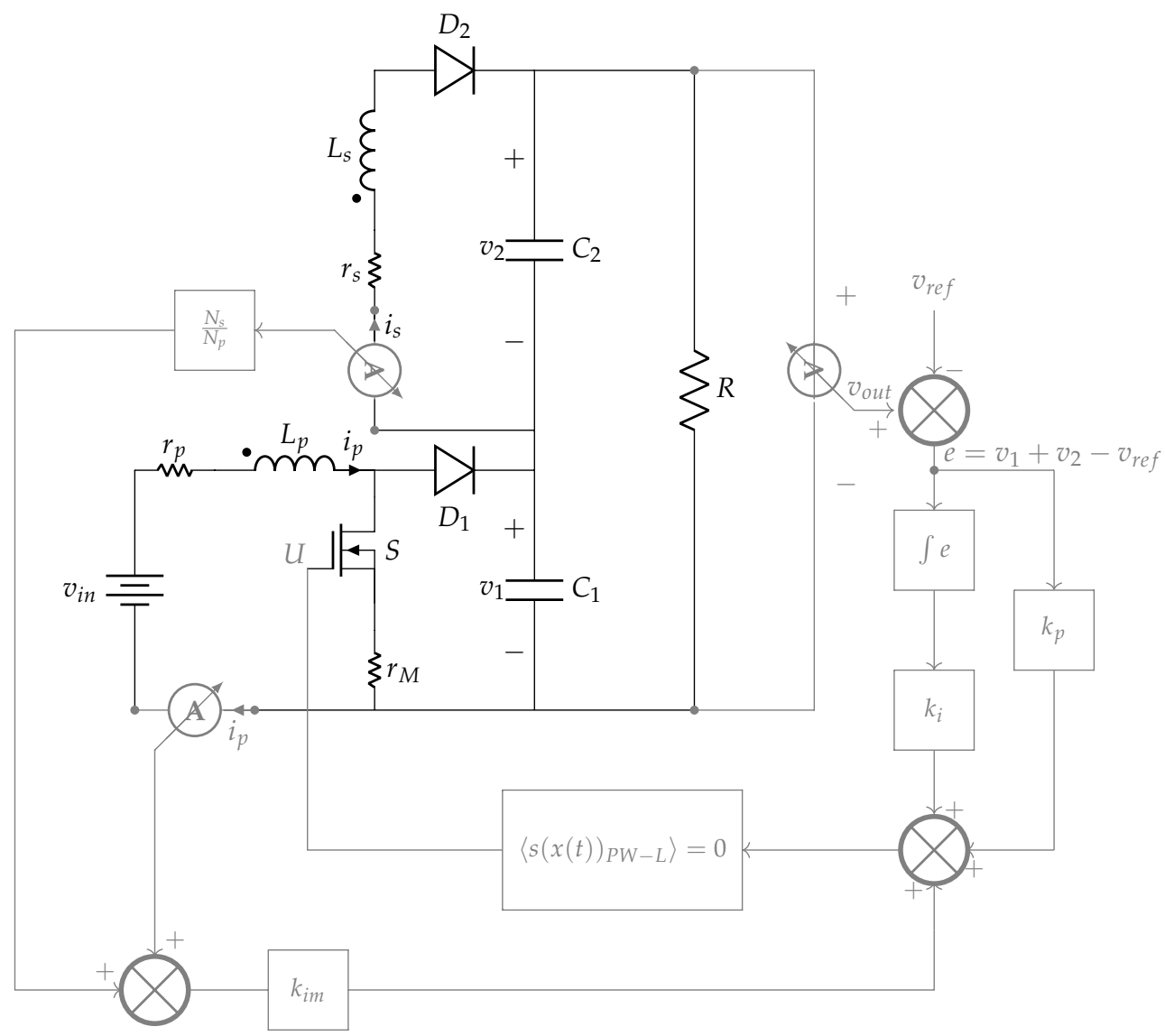

Figure 1. Boost-flyback converter with Zero Average Surface (ZAS) control.

For instance, the boost-flyback converter under peak current-mode control was analyzed in $[12,13]$ while using the complete discontinuous model. The authors took advantage of the piece-wise linear nature of the system, in order to utilize non-smooth stability assessment techniques [14-16]. These works demonstrated the transition to chaos of a periodic orbit and the importance of the slope compensation. Other control design techniques include methodologies to compute the minimum value of the compensation ramp of the peak current-mode [17], in order to enhance the stability region [18] and control the system via the hysteresis band [19]. These recently proposed methodologies emphasize the increasing importance of this power converter.

In this work, we propose applying a modified version of the Zero Average Dynamics (ZAD) control to the boost-flyback converter. The original ZAD strategy was first proposed in [20]. The main idea behind the ZAD control is to force the error of the system and its derivative to evolve as dynamical variables with zero average. To do this, the duty cycle is computed in such a way that the average condition is satisfied. In [20], a piece-wise linear approximation of the error function was proposed and successfully used in [21-24] due to the complexity to solve the equation to find the duty cycle. Unfortunately, the ZAD controller is much less robust than a regular sliding control. For this reason, here we propose controlling the system while using the same idea, i.e., we build a function $s$ that depends on the state of the system. However, as the boost-flyback is a much more complex system, we include several considerations. Firstly, we do not consider the derivative part of the error in order to avoid noise amplification in practical applications. Secondly, we include an integral control action which adds robustness to the system. Finally, we incorporate information of the currents flowing through the coils to the function $s$. Other considerations that are 
related to the behavior of the surface and its slopes remain unaltered. This modified ZAD controller is here named Zero Average Surface (ZAS) control.

The paper is organized, as follows: in Section 2, we show the mathematical model that describes the dynamics of the boost-flyback converter. Next, in Section 3, we describe the details of the ZAS control strategy when applied to the boost-flyback converter. Additionally, we show a simplified way to calculate the duty cycle by making use of the typical topological sequences that form the stable orbits in the converter. In Section 4, we analyze the stability of the orbits that result from the application of the control strategy. We perform the stability analysis under the variation of several parameters, using bifurcation diagrams and Floquet multipliers. Furthermore, in Section 5, we show the controlled system's response to a variety of disturbances, showing that the converter performs satisfactorily, despite the scale of the disturbances. Finally, in Section 6, we present some concluding remarks and point out some open problems.

\section{Dynamics of the Boost-Flyback Converter}

Figure 1 illustrates the diagram of the boost-flyback converter together with the ZAS control module. A boost-flyback converter is composed by two capacitors $C_{1}$ and $C_{2}$, two magnetically coupled coils $L_{P}$ (primary) and $L_{S}$ (secondary) with their respective internal resistances $r_{p}$ and $r_{s}$. Additionally, the system has a MOSFET $(S)$ acting as a switch with an internal resistance $r_{M}$ and two diodes $\left(D_{1}\right.$ and $\left.D_{2}\right)$. The input is provided by $v_{\text {in }}$ and the output $v_{\text {out }}$ is the sum of the voltages across capacitors $\left(v_{\text {out }}=v_{1}+v_{2}\right)$. The state of the system is then given by the voltages across the capacitors $v_{1}$ and $v_{2}$, the currents flowing through the coils $i_{P}$ and $i_{L}$, and the integral of the output error $e$. The error is, in turn, defined as the difference between the real output and the desired one, i.e., $e=v_{1}+v_{2}-v_{\text {ref }}$. Altogether, the state space $x \in \mathcal{R}^{5}$ is formed by the variables $x:=\left[\begin{array}{lllll}i_{P} & i_{S} & v_{1} & v_{2} & x_{5}\end{array}\right]^{T}$ with $x_{5}=\int e(t) d t$.

The state of the diodes and the switch can only take values in the discrete set $\{0,1\}$, i.e., each one of them can only be in an open position (no current flowing) or closed position (current is allowed to flow). Additionally, the transition between open and closed states is assumed to be instantaneous. The presence of nonlinear elements (diodes and switch) transforms the system into a non-smooth system that can be described through a set of differential equations that changes as the positions of the switch and diodes change.

Combining the different states of the diodes and the switch, there are eight topologies. However, only six of these are physically viable, which are reported in Table 1 . The primary coil $\left(L_{P}\right)$ and secondary one $\left(L_{S}\right)$ interact with each other through the mutual inductance $M=k \sqrt{L_{P} L_{S}}$, with $k \in[0,1]$ being the coupling coefficient. Additionally, we assume a small internal resistance $r_{M}$ for the switch, when it is active. When the diodes are closed, the current flow is positive and their terminal voltage is zero (ideal diodes). Conversely, when diodes are open, the terminal voltage is either negative or zero, which means that the current flow is null. In addition to the differential equations for each topology, the dynamics of the system are completed with a set of algebraic restrictions of the state variables $x$. For more details of the boost-flyback converter, we refer the reader to [10,12,13]. Finally, Table 1 summarizes the equations that describe the converter at each admissible topologies.

In Table $1, \hat{M}=L_{P} L_{S}-M^{2}$. In short hand notation, the system can be expressed as:

$$
\dot{x}(t)=f_{i}(x(t))
$$

where the subscript $i\{1,2,3,4,5,6\}$ runs through the different vector fields that are specified in Table 1 and variables are assigned as $x_{1}=i_{p}, x_{2}=i_{s}, x_{3}=v_{1}, x_{4}=v_{2}$ and $x_{5}=\int e d t$. Notice that the states $E_{1}, E_{2}, E_{3}$, and $E_{4}$ can only be present when the MOSFET is inactive $(\mathrm{OFF})$, and states $E_{5}$ and $E_{6}$ when the MOSFET is active $(\mathrm{ON})$. 
Table 1. Differential equations describing the six admissible topologies denoted as $E_{i}$ with $i=\{1,2,3,4,5,6\}$.

\begin{tabular}{|c|c|}
\hline$S=0, D_{1}=0$ and $D_{2}=0$ & $S=0, D_{1}=1$ and $D_{2}=0$ \\
\hline$E_{1}:=\left\{\begin{array}{l}\dot{i}_{P}=0 \\
\dot{i}_{S}=0 \\
\dot{v}_{1}=-\left(v_{1}+v_{2}\right) /\left(R C_{1}\right) \\
\dot{v}_{2}=-\left(v_{1}+v_{2}\right) /\left(R C_{2}\right) \\
\dot{x}_{5}=v_{1}+v_{2}-v_{\text {ref }}\end{array}\right.$ & $E_{2}:=\left\{\begin{array}{l}\dot{i}_{P}=-\left(r_{P} i_{P}+v_{1}-v_{i n}\right) / L_{P} \\
\dot{i}_{S}=0 \\
\dot{v}_{1}=\left(R i_{P}-v_{1}-v_{2}\right) /\left(R C_{1}\right) \\
\dot{v}_{2}=-\left(v_{1}+v_{2}\right) /\left(R C_{2}\right) \\
\dot{x}_{5}=v_{1}+v_{2}-v_{r e f}\end{array}\right.$ \\
\hline$S=0, D_{1}=0$ and $D_{2}=1$ & $S=0, D_{1}=1$ and $D_{2}=1$ \\
\hline$E_{3}:=\left\{\begin{array}{l}\dot{i}_{P}=0 \\
\dot{i}_{S}=-\left(r_{S} i_{S}+v_{2}\right) / L_{S} \\
\dot{v}_{1}=-\left(v_{1}+v_{2}\right) /\left(R C_{1}\right) \\
\dot{v}_{2}=\left(R i_{S}-v_{1}-v_{2}\right) /\left(R C_{2}\right) \\
\dot{x}_{5}=v_{1}+v_{2}-v_{r e f}\end{array}\right.$ & $E_{4}:=\left\{\begin{array}{l}\dot{i}_{P}=\left(M r_{S} i_{S}-r_{P} L_{S} i_{P}+L_{S}\left(v_{i n}-v_{1}\right)+M v_{2}\right) / \hat{M} \\
\dot{i}_{S}=\left(M r_{P} i_{P}-r_{S} L_{P} i_{S}+M\left(v_{1}-v_{i n}\right)-L_{P} v_{2}\right) / \hat{M} \\
\dot{v}_{1}=\left(R i_{P}-v_{1}-v_{2}\right) /\left(R C_{1}\right) \\
\dot{v}_{2}=\left(R i_{S}-v_{1}-v_{2}\right) /\left(R C_{2}\right) \\
\dot{x}_{5}=v_{1}+v_{2}-v_{r e f}\end{array}\right.$ \\
\hline$S=1, D_{1}=0$ and $D_{2}=0$ & $S=1, D_{1}=1$ and $D_{2}=1$ \\
\hline$E_{5}:=\left\{\begin{array}{l}\dot{i}_{P}=\left(v_{i n}-\left(r_{P}+r_{M}\right) i_{P}\right) / L_{P} \\
\dot{i}_{S}=0 \\
\dot{v}_{1}=-\left(v_{1}+v_{2}\right) /\left(R C_{1}\right) \\
\dot{v}_{2}=-\left(v_{1}+v_{2}\right) /\left(R C_{2}\right) \\
\dot{x}_{5}=v_{1}+v_{2}-v_{\text {ref }}\end{array}\right.$ & $E_{6}:=\left\{\begin{array}{l}\dot{i}_{P}=\left(M r_{S} i_{S}-\left(r_{P}+r_{M}\right) L_{S} i_{P}+M v_{2}+L_{S} v_{i n}\right) / \hat{M} \\
\dot{i}_{S}=\left(M\left(r_{P}+r_{M}\right) i_{P}-r_{S} L_{P} i_{S}-L_{P} v_{2}-M v_{i n}\right) / \hat{M} \\
\dot{v}_{1}=-\left(v_{1}+v_{2}\right) /\left(R C_{1}\right) \\
\dot{v}_{2}=\left(R i_{S}-v_{1}-v_{2}\right) /\left(R C_{2}\right) \\
\dot{x}_{5}=v_{1}+v_{2}-v_{r e f}\end{array}\right.$ \\
\hline
\end{tabular}

\section{Zero Average Surface Control Technique}

Pulse Width Modulation (PWM) is a widely used methodology for controlling the output voltage in a power converter [25]. In a PWM, the MOSFET commutes between the OFF position $(u=0)$ and the ON position $(u=1)$ in order to regulate the system's output. For the boost-flyback converter, the more time the discrete signal remains in the ON position, the higher output voltage. In order to obtain the desired output voltage value, it is necessary to compute the duty cycle $(d)$, i.e., the ratio between the time that the signal $u=1$ and period $T$ of the MOSFET, namely $d=\frac{\left.t\right|_{u=1}}{T}$. In summary, the duty cyle is the key quantity that one needs to control in a PWM based power converter. In this section, we present a simple methodology to calculate $d$ at each switching cycle, called Zero Average Surface control.

\subsection{Defining the Surface $s(x(t))$}

Briefly speaking, we calculate the duty cycle based on the idea that a certain statedependent function $s(x(t))$ has a zero average in a PWM cycle. The function should include the most information regarding the system's state. This idea is inspired by a previously reported technique, named the Zero Average Dynamics (ZAD) strategy, which was originally applied to the buck power converter [20,26]. In these works, the function $s(x(t))$ was defined as a linear combination between the output error and its derivative. However, the absence of an integral control rendered the robustness of the system unsatisfactory. In this work, we define the function $s(x(t))$ via a strategically selected combination between the five states of the boost-flyback: The voltages across the capacitors are linked through the desired output voltage. Additionally, the currents through the coils are linked via the magnetization current. Finally, an integral control action is also added, such that the function reads

$$
s(x(t))=k_{p}\left(x_{3}+x_{4}-v_{r e f}\right)+k_{i} x_{5} d t+k_{i m}\left(x_{1}+\frac{N s}{N p} x_{2}\right)
$$

Because $v_{\text {out }}=x_{3}+x_{4}$ supplies the desired output voltage $v_{\text {ref }}$ (see Figure 1 ), then $k_{p}\left(x_{3}+x_{4}-v_{r e f}\right)$ is a proportional control action. Furthermore, the integral control is $k_{i} x_{5}$, where $x_{5}(t)=\int\left(x_{3}+x_{4}-v_{r e f}\right) d t$. These two terms define a PI controller that reduces 
the steady state output error and handles the transient state when the system is subject to disturbances and/or changes in the system's parameters. The current values $x_{1}$ and $x_{2}$, enter into the expression via the magnetization current $x_{1}+N_{s} / N_{p} x_{2}$ [27], where $N_{p}$ $\left(N_{S}\right)$ is the number of turns in the primary (secondary) coil. This last ingredient of the expression acts as an additional proportional control and its role is regulated with $k_{i m}$.

\subsection{Piece-Wise Linear Approximation of the Function}

The control signal $u$ in a centered PWM (see upper part of Figure 2) can be defined as:

$$
u=\left\{\begin{array}{lll}
1 & \text { if } \quad k T \leq t \leq k T+T \frac{d}{2} \\
0 & \text { if } \quad k T+T \frac{d}{2}<t<(k+1) T-T \frac{d}{2} \\
1 & \text { if } \quad(k+1) T-T \frac{d}{2} \leq t \leq(k+1) T
\end{array}\right.
$$

where $T=50 \mu$ s is the sampling period and $d \in[0,1]$ is the duty cycle. As previously mentioned, the aim of the controller is to compute the duty cycle $d$ in order to achieve, in every $T$-cycle, a zero average of the function that is defined by (2), i.e.,

$$
\langle s(x(t))\rangle_{T}=\left\langle k_{p}\left(x_{3}+x_{4}-v_{r e f}\right)+k_{i} x_{5} d t+k_{i m}\left(x_{1}+\frac{N s}{N p} x_{2}\right)\right\rangle_{T}=0
$$

In this work, a centered PWM is used to compute the duty cycle in every T-cycle, such that Equation (4) meets the zero average criterion. Equation (4) can be written as:

$$
\int_{k T}^{(k+1) T} s(x(t)) d t=0
$$

Solving this equation implies computing the zeros of a complicated transcendental equation, which is highly inefficient from a practical point of view. In order to overcome this problem, we assume that the surface $s(x(t))$ can be approximated via a piece-wise linear function in time $s(x(t))_{P W-L}$, forming a triangle-like wave (see lower part of Figure 2). Secondly, we will assume that the derivatives of the first and third segments of $s(x(t))_{P W-L}$ are equal, and, thirdly, we suppose that we can calculate the slopes of the piece-wise linearly approximated function at the beginning of the cycle (denoted as $k T$ ). In summary, $s(x(t))_{P W-L}$ can be expressed as:

$$
s(x(t))_{P W-L}=\left\{\begin{array}{lll}
\dot{s}_{1}(x(k T)) t+s_{1}(x(k T)) & \text { if } \quad k T \leq t \leq k T+T \frac{d}{2} \\
\dot{s}_{2}(x(k T)) t+s_{2}(x(k T)) & \text { if } \quad k T+T \frac{d}{2}<t<(k+1) T-T \frac{d}{2} \\
\dot{s}_{1}(x(k T)) t+s_{3}(x(k T)) & \text { if } \quad(k+1) T-T \frac{d}{2} \leq t<(k+1) T
\end{array}\right.
$$

Here, $s_{1}(x(k T)), s_{2}(x(k T))$ and $s_{3}(x(k T))$ are the offset values of the three segments (see Figure 2). Similarly, $\dot{s}_{1}(x(k T))$ and $\dot{s}_{2}(x(k T))$ are the slopes that are computed from the expression of $\dot{s}(x(t))$ while using the state values at the beginning of the cycle. These derivatives can be written in the following form:

$$
\begin{aligned}
& \dot{s}_{1}(x(k T))=\frac{\partial s}{\partial x} f_{i} \\
& \dot{s}_{2}(x(k T))=\frac{\partial s}{\partial x} f_{j}
\end{aligned}
$$

where the subscript $i$ can take the values $i=\{5,6\}$, when considering that $E_{5}$ and $E_{6}$ are the two admissible topologies with MOSFET in active mode. Similarly, the subscript $j$ can take the values $j=\{1,2,3,4\}$, which is consistent with the fact that the admissible states with the MOSFET in inactive mode are $E_{1}, E_{2}, E_{3}$, and $E_{4}$. In the following section, we will describe the topologies that are to be used to compute $\dot{s}_{1}\left(x(k T)\right.$ and $\dot{s}_{2}(x(k T)$. 


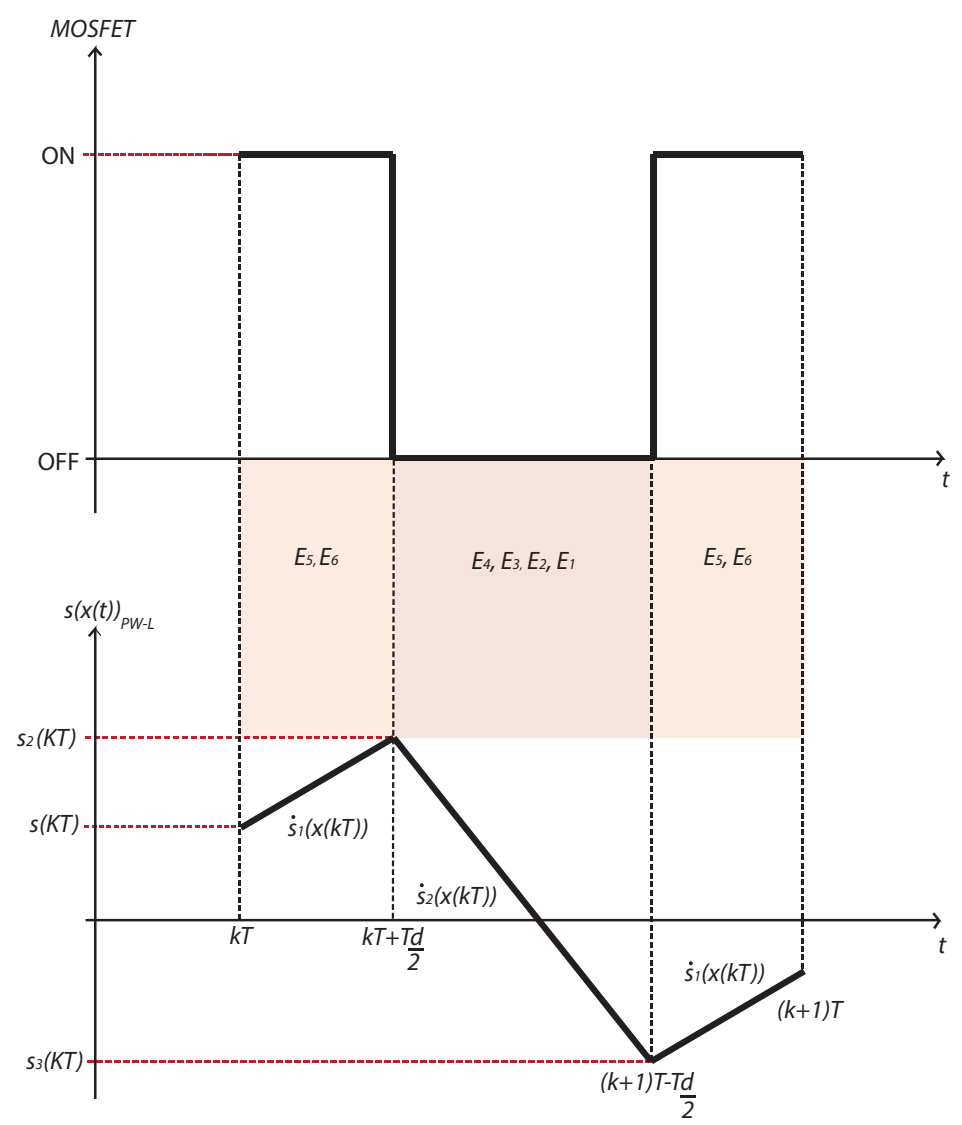

Figure 2. Schematic diagram of the approximated Zero Average Dynamics (ZAD) function. Upper panel shows the two positions of the MOSFET (ON and OFF) with the the corresponding piece-wise linear approximation of the function $s(x(t))_{P W-L}$. When the MOSFET position is ON, topologies $E_{5}$ and $E_{6}$ are physically feasible. Similarly, with MOSFET position OFF, only $E_{1}, E_{2}, E_{3}$ and $E_{4}$ are the admissible topologies.

\subsection{Computing the Duty Cycle}

A lot of analytical and numerical results $[10,12,13,17-19]$ have shown that there are three topological sequences that form different period-1 orbits, depending on the system's parameters: (i) $E_{5} \rightarrow E_{4} \rightarrow E_{3} \rightarrow E_{6}$, (ii) $E_{5} \rightarrow E_{4} \rightarrow E_{3} \rightarrow E_{1}$, and (iii) $E_{5} \rightarrow E_{4} \rightarrow E_{6}$. Figure 3 shows the three basic possible period- 1 orbits and their topologies. According to these orbits, we can draw the following conclusions: (1) out of the topologies appearing with the MOSFET in active mode ( $E_{5}$ and $\left.E_{6}\right), E_{5}$ is always present. Similarly, for topologies with the MOSFET in the inactive state $\left(E_{4}, E_{3}\right.$, and $\left.E_{1}\right) E_{4}$ is always present. (2) The power converter always switches the MOSFET position between states $E_{5}$ and $E_{4}$. These two facts lead us to conclude that the main part of the sequence that describes any periodic orbit is $E_{5} \rightarrow E_{4}$. Subsequently, to apply the approximation (6), and taking into account Equation (7), we can describe any sequence as $E_{5}$, followed by $E_{4}$. This implies that $\dot{s}_{1}$ is computed with topology $E_{5}$ and $\dot{s}_{2}$ is computed with topology $E_{4}$. Under this consideration, the orbits that the system can exhibit are $E_{5} \rightarrow E_{4} \rightarrow E_{3} \rightarrow E_{6}$ (5436 for short, see Figure 3A), $E_{5} \rightarrow E_{4} \rightarrow E_{3} \rightarrow E_{1}$ (5431, see Figure 3B), and $E_{5} \rightarrow E_{4} \rightarrow E_{6}$ (546, see Figure 3C).

Particularly, in every cycle, we will assume that $E_{5}$ is present for a time $t \in[k T, k T+$ $T d / 2]$, and, at $t=k T+T d / 2$, the system commutes to $E_{4}$. Moreover, when the MOSFET is inactive, i.e during the interval $t \in[k T+T d / 2,(k+1) T-T d / 2]$, the system may undergo one or two secondary transitions: (i) the first one will appear when $i_{P}=0$, and, in this case topology, $E_{3}$ guides the system and (ii) the second one will appear when $i_{S}=0$ and $E_{1}$ guide the dynamics. Finally, for the interval $t \in[(k+1) T-T d / 2,(k+1) T]$, the system will switch its dynamics to $E_{6}$ in the cases in which $i_{S} \neq 0$ and it will rapidly evolve $i_{S}$ 
towards 0 , where the $E_{5}$ topology leads the dynamics of the system. Conversely, when the previous topology in inactive state was in $E_{1}$, it will immediately switch its behavior to $E_{5}$.

A

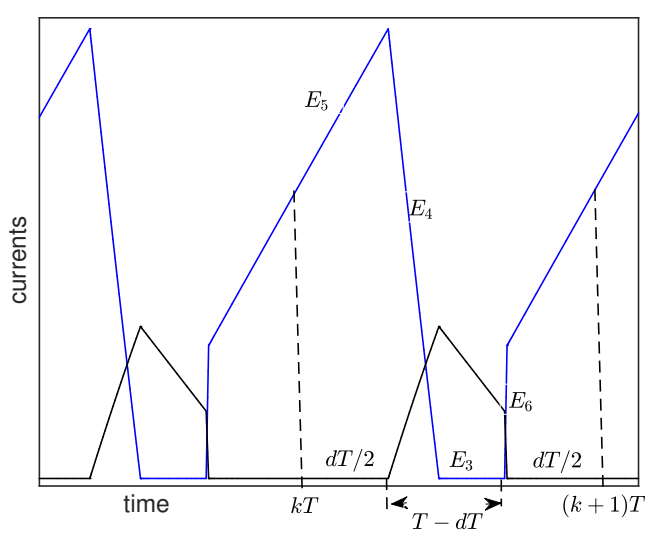

B

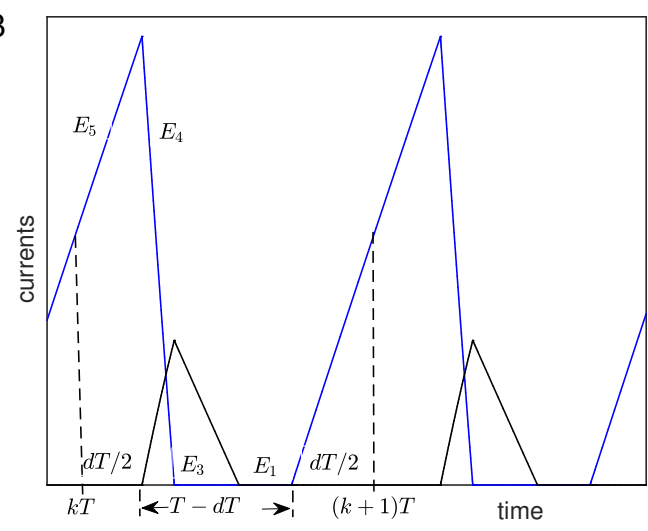

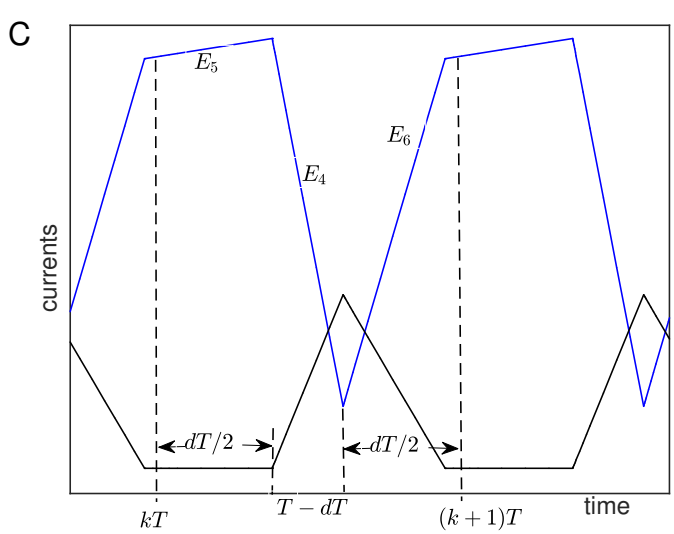

Figure 3. Three possible periodic orbits in the boost-flyback converter. (A) Orbit following the topologies 5436. (B) Orbit 5431 and (C) Orbit 546. In all panels, the primary (secondary) current $i_{P}\left(i_{S}\right)$ is depicted in blue (black).

According to the previous considerations, $\dot{s}_{1}(x(k T))$ must be evaluated with $E_{5}$ and $\dot{s}_{2}(x(k T))$ must be evaluated with $E_{4}$. Additionally, the offset of each one of the segments can be computed as:

$$
s_{2}(x(k T))=s_{1}(x(k T))+\frac{d T}{2} \dot{s}_{1}(x(k T))
$$

and

$$
s_{3}(x(k T))=s_{1}(x(k T))+\frac{d T}{2} \dot{s}_{1}(x(k T))+(T-d T) \dot{s}_{2}(x(k T))
$$

where $s_{1}(x(k T))$ is the value of the $s(x(t))$ at the beginning of the cycle. With these assumptions, Equation (5) is simplified in such a way that an algebraic equation is solved instead of a transcendental one. The simplified equation now reads as:

$$
\int_{k T}^{(k+1) T} s(x(t))_{P W-L} d t=0
$$

After some straightforward algebra, the duty cycle $d$ can be solved as:

$$
d=\frac{2 s_{1}(x(k T))+T \dot{s}_{2}(x(k T))}{T\left(\dot{s}_{2}(x(k T))-\dot{s}_{1}(x(k T))\right)}
$$


Finally, because the duty cycle $d_{k}$ to apply at the $k T$-cycle must be bounded within the range $[0,1]$, we complement the duty cycle calculation with the following saturating conditions:

$$
d_{k}:=\left\{\begin{array}{lll}
0 & \text { if } & d<0 \\
d & \text { if } & 0 \leq d \leq 1 \\
1 & \text { if } & d>1
\end{array}\right.
$$

Altogether, the design of the proposed controller can be summarized, as follows:

1. Define a sampling time $T$ of the MOSFET according to design requirements.

2. Define the surface $s(x(t))$, including the information of: (i) the states of the system and (ii) the integral of error to correct steady state error (Equation (2)).

3. Apply the piece-wise linear approximation $s(x(t))_{P W-L}$ of the surface (Equation (6)).

4. Sense the output voltage error, magnetization current, and integral of error at the beginning of every cycle.

5. Compute, at the beginning of every cycle, the duty cycle that ensures $\int_{0}^{T} s(x(t))_{P W-L} d t=0$ (Equation (9)).

6. Apply the computed duty cycle (Equations (10) and (11)).

\subsection{Stability Analysis of the Period-1 Orbits}

With the aim of assessing the stability of the period-1 orbits, we proceed to calculate the associated Floquet multipliers $\lambda$. Floquet multipliers are the eigenvalues of the monodromy matrix $\mathcal{M}$, which describes the evolution of an infinitesimal perturbation $\delta x$ around the target periodic orbit from one period to another, namely

$$
\delta x((k+1) T)=\mathcal{M} \delta x(k T)
$$

In the case of smooth systems, computing $\mathcal{M}$ can be done straightforwardly via the linearization of the vector field. Conversely, the formal calculation of monodromy matrices in non-smooth systems requires appropriate corrections that are induced by the transitions between topologies, which are described by saltation matrices [28,29]. Here, we will make use of a much simpler numerical approach to the calculation of $\mathcal{M}$, which only requires the evolution of a reasonable number of perturbed orbits between two periods. Because our system is non-autonomous, the periodic orbit is stable if all eigenvalues lie within the unit circle [30]. The algorithm can be summarized as (see [31] for details):

1. Find the periodic orbit $x^{*}$ and the associated duty cycle $d$, which guarantees that $x^{*}(k T)=x^{*}((k+1) T)$ and identify the topological sequence that fulfils the condition.

2. Create $N$ randomly perturbed orbits $x_{p}(k T)=x^{*}(k T)+\epsilon$ with $\|\epsilon\| \approx \mathcal{O}\left(10^{-4}\right)$.

3. Evolve the perturbed orbits over a period $T$, such that $y_{p}=x_{p}((k+1) T)$.

4. Store the differences of every state between the perturbed and the unperturbed orbits at the beginning of the period $(\Delta x)$ and at the end of it $(\Delta y)$.

5. Approximate the monodromy matrix as:

$$
\mathcal{M}=\left[\left(\Delta x^{T} \Delta x\right)^{-1} \Delta x^{T} \Delta y\right]
$$

6. Compute the Floquet multipliers via the eigenvalues $\lambda_{i}$ of $\mathcal{M}$.

\section{Numerical Results}

We proceed to set the values of the constants $k_{p}, k_{i}$, and $k_{i m}$ in order to guarantee the stability of the orbit. It can be seen that the controller includes two loops: the first one is external and it corresponds to the voltage control loop, which is formed by the control action that is based on the error and its integral. This loop guarantees that the output voltage follows the reference value. For this reason, $k_{i}$ should be high in order to obtain a fast dynamic response. Conversely, as the proportional action controlled by $k_{p}$ is more sensitive to errors, it should not be high in order to avoid instabilities. The second loop, corresponding to the proportional control of the magnetization current, is an inner loop, and it provides 
stability to the system, and, following the same guidelines as before, it should not have a high value. For simulations, we set the constant values $k_{p}$ and $k_{i}$ close to the values that were reported in $[12,13]$. In turn, $k_{i m}$ has been set, such that a stable period-1 orbit with topological sequence 5436 is obtained, which we have selected as the target periodic orbit.

In what follows, we investigate the stability of the period-1 orbit that was described by the sequence 5436 using bifurcation diagrams and Floquet multipliers. Several works have demonstrated that this orbit is very stable [12,17-19]. In this section, only the variation of the parameters $R, v_{i n}$, and $v_{r e f}$ are analyzed, such that $k_{i}, k_{i m}$, and $k_{p}$ remain fixed. In Table 2 , all of the parameter values are presented. The nominal values of $R, v_{i n}$, and $v_{\text {ref }}$ are identified with the subscript $N$ and the range of variation used in the stability analysis is shown in front.

Table 2. The parameter values used in simulation. $v_{r e f}, v_{i n}$, and $R$ are used for bifurcation diagrams and simulating disturbances. Unless otherwise stated, the nominal values $v_{i n, N}, v_{r e f, N}$, and $R_{N}$ are used.

\begin{tabular}{cc}
\hline \multicolumn{3}{c}{ Parameter Values } \\
\hline$v_{i n, N}=18 \mathrm{~V}$ & $v_{\text {in }} \in[6,18] \mathrm{V}$ \\
\hline$v_{r e f, N}=100 \mathrm{~V}$ & $v_{\text {ref }} \in[80,280] \mathrm{V}$ \\
\hline$R_{N}=200 \Omega$ & $R \in[28,800] \Omega$ \\
\hline$L_{P}=240.3 \mu \mathrm{H}$ & $L_{S}=816.2 \mu \mathrm{H}$ \\
\hline$C_{1}=220 \mu \mathrm{F}$ & $C_{2}=220 \mu \mathrm{F}$ \\
\hline$\frac{N_{s}}{N_{p}}=\sqrt{L_{s} / L_{p}}$ & $k=0.96$ \\
\hline$r_{P}=0.02 \Omega$ & $r_{S}=0.3 \Omega$ \\
\hline$r_{M}=0.044$ & $k_{p}=1.5 \mathrm{~V}^{-1}$ \\
\hline$k_{i}=350[\mathrm{~V} \cdots]^{-1}$ & $k_{i m}=3 \mathrm{~A}^{-1}$ \\
\hline
\end{tabular}

Let us start by analyzing the behavior of the system under variations in the input voltage $v_{\text {in }}$. In Figure 4, two different protocols are presented. The red continuous curve corresponds to increasing variations of the parameter, while the blue empty symbols refer to decreasing variations of the parameter. On the one hand, Figure $4 \mathrm{~A}$ shows that, by increasing $v_{i n}$, a period- 2 orbit shows up as signaled by the presence of two values of the duty cycle. This 2-periodic orbit is characterized by one saturated cycle, which remains stable for almost the whole range of the parameter variation until it suddenly transforms into a period- 1 solution at $v_{\text {in }} \approx 17.8 \mathrm{~V}$. Interestingly, the same analysis performed starting with a high value of $v_{i n}$ reveals the co-existence of a stable period- 1 orbit with the saturated solution that was described before. The period- 1 solution remains stable until $v_{\text {in }} \approx 6.9$, where it bifurcates to an unsaturated period- 2 orbit, which eventually merges at $v_{\text {in }} \approx 6.4$ with the saturated period- 2 orbit found for increasing $v_{i n}$. The period- 1 orbit corresponds to the topological sequence 5436 and Figure 4B depicts the norm of largest eigenvalue $\lambda_{\text {max }}$ of the monodromy matrix that is associated with this orbit (filled black symbols). By looking at $\lambda_{\max }$, one can see that the stability is lost $\left(\left|\lambda_{\max }\right|>1\right)$ at $v_{\text {in }} \approx 6.9$, i.e, at the bifurcation point. It shall be noticed that, at smaller values of $v_{i n}$, there indeed exists a period- 1 orbit that is nonetheless unstable (empty symbols). It is worth noticing that the region in which stability of the desired orbit is lost $\left(s m a l l v_{i n}\right)$ corresponds to high gains of the converter. In this scenario, the current requirements are high in the primary coil. This observation helps to understand the mechanism of the bifurcation: as the duty cycle increases, the current flowing through the primary coil increases. Therefore, when the switch is turned OFF and topology $E_{4}$ kicks in, $i_{P}$ cannot evolve towards 0 before turning the switch $\mathrm{ON}$ again. The consequence is that topology $E_{3}$ is never reached and the orbit evolves as 546 during one cycle (upper branch of the unsaturated period-2 orbit). In the next cycle, the 5436 is recovered, because the initial condition of the primary current is now 
at a much lower value, allowing for $i_{P}$ to reach zero value when the MOSFET is OFF. Finally, the upper branch eventually saturates to $d=1$, which indicates that, during one whole period, the system remains in topology $E_{5}$. Meanwhile, in the next cycle, the sequence 5436 is recovered.

Figure $4 \mathrm{C}$ depicts the output error that is produced by the coexisting orbits previously described. Here, one can see that the output error in the two sampled points of the period-2 orbits are quite similar, producing the thick lower line. Similarly, the error that is associated to the period 1 orbit, while it has a larger absolute value, remains within the reasonable range $<0.25 \%$.
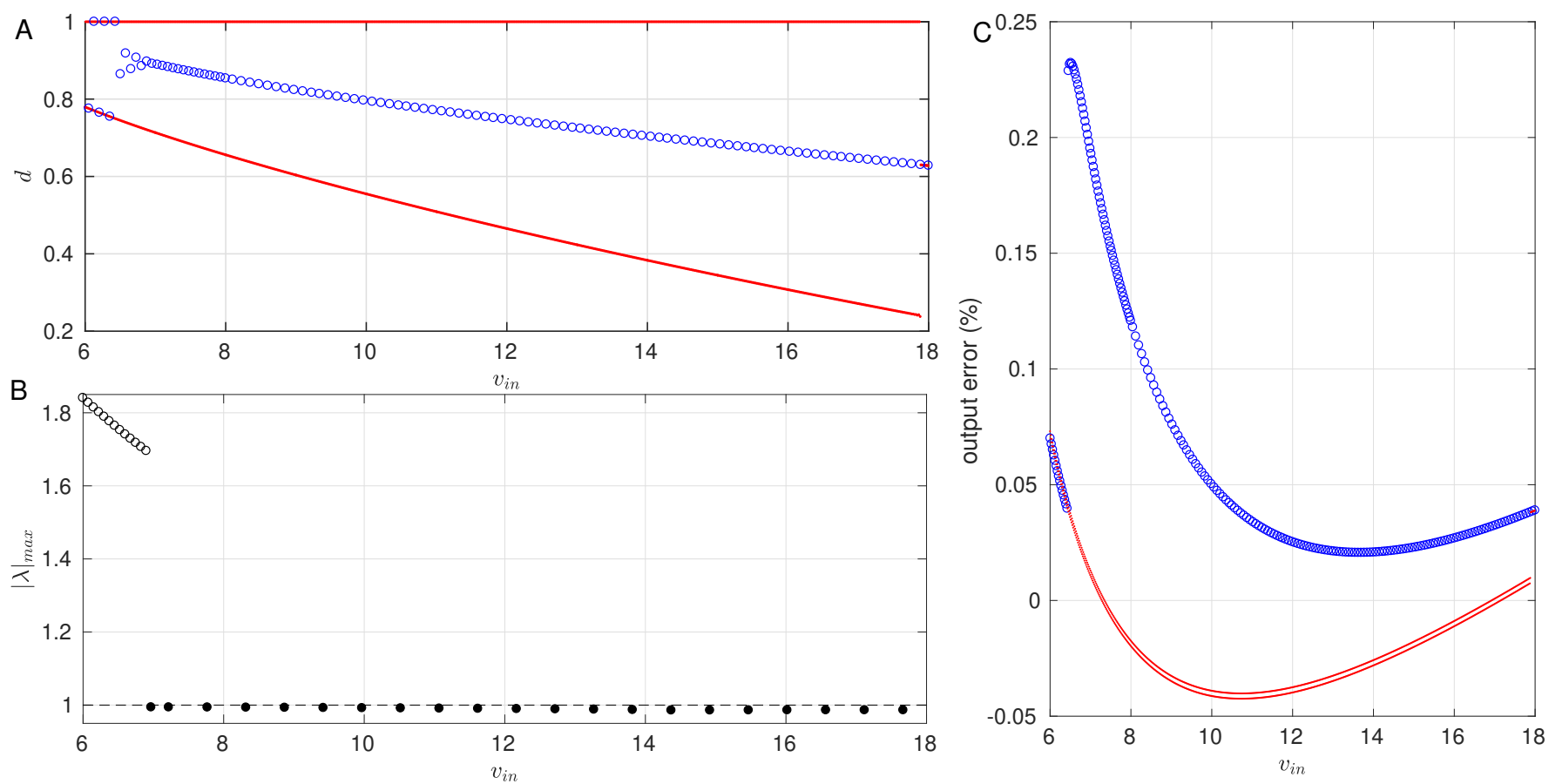

Figure 4. Bifurcations with $v_{\text {in }}$ as a control parameter. (A). Duty cycle solution (11) in steady state with two protocols: Red (blue) line corresponds to increasing (decreasing) variations of the parameter. (B). Maximum Floquet multiplier of the period- 1 orbits found in the studied interval. Solid (empty) symbols refer to stable (unstable) orbits. (C). Output error of the attractors of panel (A), with the same color code. Parameters of this simulation, as in Table 2.

Next, we focused our attention on the bifurcations that arise by varying the reference voltage $v_{r e f}$ with the same protocols described before. In Figure 5A, we show the values of the solution of the duty cycle by increasing (red) and decreasing (blue) the reference voltage $v_{r e f}$. Following the increasing bifurcation curve, it is possible to see that the period1 orbit 5436 is stable over a large range of $v_{r e f} \in[80,252]$. After this point, the orbit bifurcates to the unsaturated period-2 orbit until it finally reaches the saturated period-2 orbit. As described in $v_{\text {in }}$ variation, the bifurcation mechanism is the same: the bifurcation is produced by the high $i_{P}$ requirements, which leads to increasing values of the duty cycle. Figure 5B supports these findings, i.e, the norm of the largest Floquet exponent of the period-1 orbit is $\left|\lambda_{\max }\right|<1$ over the same range of stability that is reported above. Once again, after the bifurcation point, a period- 1 orbit still exists with unstable nature.

Following the decreasing parameter protocol, one starts with the saturated 2-period cycle, which persists until $v_{r e f} \approx 120 \mathrm{~V}$, indicating a bistable behavior in that interval. Below this value, the saturated period-2 orbit disappears and the only attractor is the period- 1 cycle.

Additionally, Figure 5C displays the output error using the increasing and decreasing protocols, where it can be noticed that the period- 1 orbit presents an almost negligible error $(<0.02 \%)$ that rapidly increases after the bifurcation. Nevertheless, even the period-2 orbits (saturated and unsaturated) are associated with reasonably small values of the error $(<0.2 \%)$. 

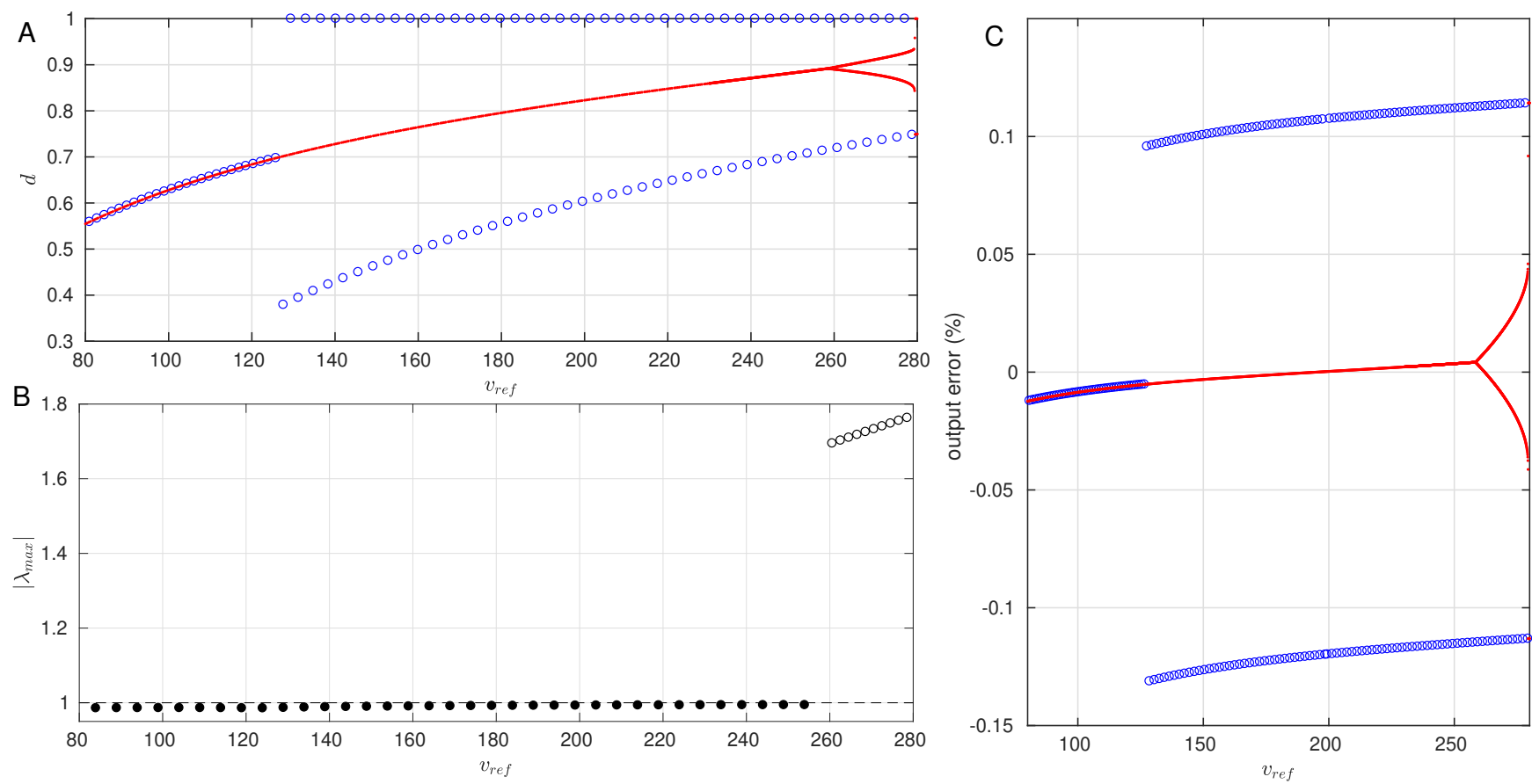

Figure 5. Bifurcations with $v_{r e f}$ as a control parameter. (A). The duty cycle solution (11) in steady state with two protocols: Red (blue) line corresponds to increasing (decreasing) variations of the parameter. (B). Maximum Floquet multiplier of the period- 1 orbits found in the studied interval. Solid (empty) symbols refer to stable (unstable) orbits. (C). Output error of the attractors of panel A, with the same color code. The parameters of this simulation, as in Table 2.

Finally we will focus our attention on the bifurcations with varying load $(R)$, as displayed in Figure 6. It is useful to start with the decreasing protocol, where a period-1 solution is present in the interval $R \in[658,800] \Omega$. This periodic solution corresponds to the topological sequence 5431 . This orbit is different from the orbit 5436 that we have studied so far. This sequence appears when the duty cycle is small and, therefore, the MOSFET remains in the OFF position for a long time. During this OFF time, the current through the secondary coil $i_{S}$ has enough time to reach a zero value. This means that topology $E_{1}$ guides the dynamics for the rest of the OFF interval. Once the switch is active again, topology $E_{5}$ immediately kicks in without the intermediate topology $E_{6}$. The stability of the 5431 orbit is corroborated with the values of $\left|\lambda_{\max }\right|<1$ in the same interval (solid circles in Figure 6B). At $R<658 \Omega$, the usual 5436 orbit is recovered as the duty cycle increases. The orbit remains stable until a rapid bifurcation to the period- 2 solution at $R \approx 35 \Omega$ and the subsequent saturation. This can be also observed by the stable interval that is characterized by $\left|\lambda_{\max }\right|<1$ of the 5436 orbit (solid squares) in Figure 6B. As expected, it changes stability $\left(\left|\lambda_{\max }\right|>1\right)$ at the bifurcation point (empty circles).

Similarly, by increasing $R$, one starts with the saturated period-2 orbit. It remains stable until $R \approx 150 \Omega$, where a chaotic attractor emerges in a small window. This attractor disappears and gives rise to the stable period- 1 sequence 5436 at $R>185 \Omega$. As in the previous cases, the associated errors of the period 1 orbit are small, as illustrated in Figure $6 \mathrm{C}$, and it only starts increasing at the bifurcations. Despite this, the period-2 orbits also display small values of the error. 

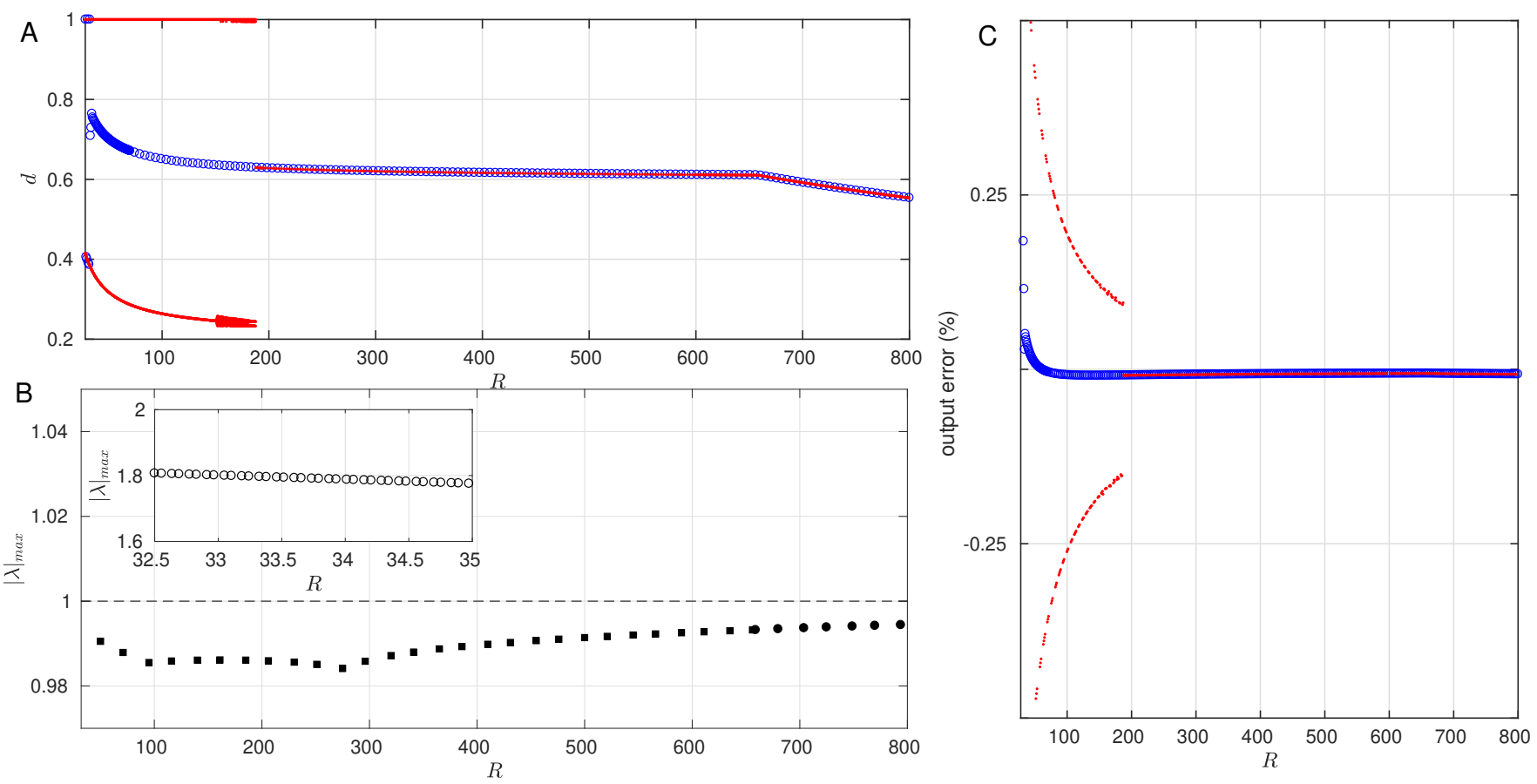

Figure 6. Bifurcations with $R$ as a control parameter. (A). Duty cycle solution (11) in steady state with two protocols: Red (blue) line corresponds to increasing (decreasing) variations of the parameter. (B). Maximum Floquet multiplier of the period- 1 orbits found in the studied interval. Solid (empty) symbols refer to stable (unstable) orbits. (C). Output error of the attractors of panel A, with the same color code. The parameters of this simulation, as in Table 2.

\section{Disturbances Rejection}

So far we have shown the co-existence of the desired period- 1 orbit with a period-2 solution over a large interval of the studied parameters. In this section, we test the robustness of the orbit 5436 when different disturbances are present in the system. The disturbances that are simulated in this part correspond to abrupt changes in the parameter values, which were used as bifurcation parameters.

Firstly, in Figure 7A, we show the convergence of the system's output from an initial value of $80 \mathrm{~V}$ to a desired value of $v_{r e f}=100 \mathrm{~V}$. It can be seen that the system rapidly establishes to the steady state value in around $t \approx 10 \mathrm{~ms}$. After this, a sudden change in the desired output from $v_{r e f}=100 \mathrm{~V}$ to $v_{r e f}=80 \mathrm{~V}$ is performed at $t=30 \mathrm{~ms}$ and the system is able to reach the new desired value at around $t \approx 50 \mathrm{~ms}$. In Figure 7B, we show the evolution of the output error to the disturbances that were described before, together with the evolution of the duty cycle and a sample trace of the function $s(x(t))$ in a small time interval. In the case of the desired period 1 orbit, the duty cycle transiently saturates, but it eventually reaches the asymptotic $d$-value corresponding to the stable orbit (see insets). Moreover, one can also notice that the piece-wise linear assumption of $s(x(t))$ in Equation (6) is adequate, as testified by the virtually piece-wise linear nature of the function that is defined in Equation (2). 

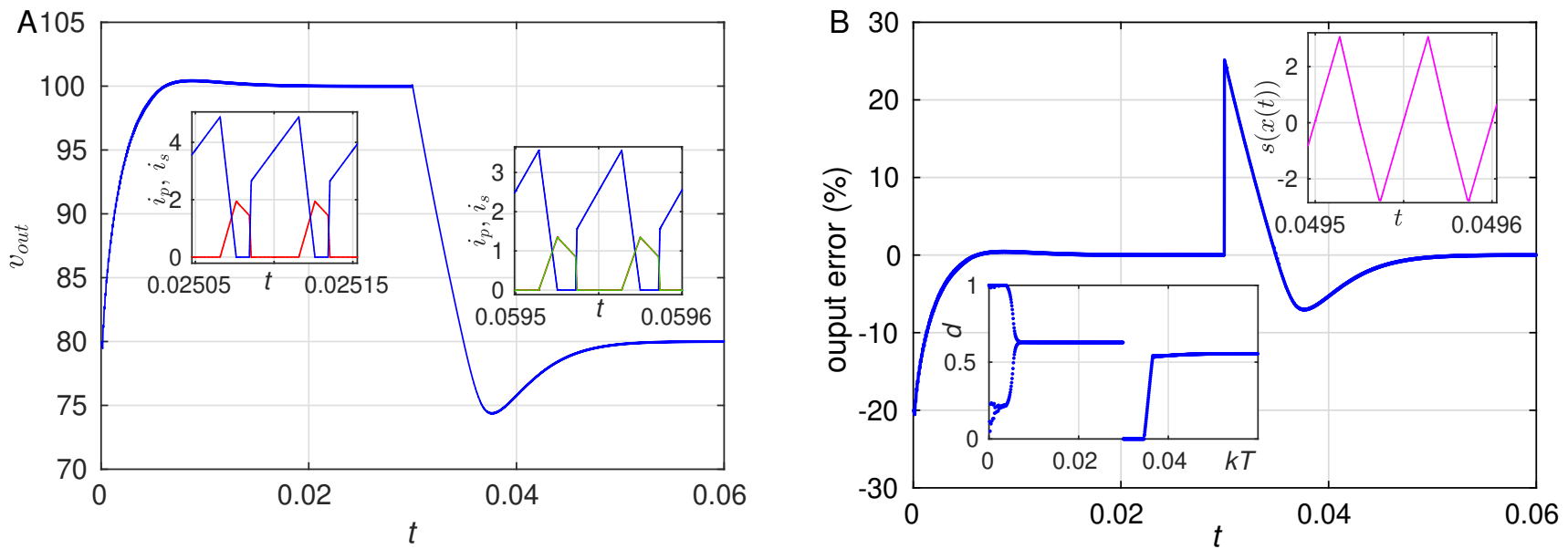

Figure 7. (A). Output voltage as a function of time with a disturbance in the reference voltage presented at $t=0.03 \mathrm{~s}$. Insets: primary (secondary) currents are depicted in blue (red) before and after the change in $v_{\text {out }}$. (B). Output error as a function of time for the evolution of $v_{\text {out }}$ in panel (A). Lower inset: dynamics of the duty cycle. Upper inset: function $s(x(t))$ defined in Equation (2). In this figure, the parameters have been chosen, as in Table 2, with a reference variation from $v_{r e f}=100 \mathrm{~V}$ to $v_{\text {ref }}=80 \mathrm{~V}$.

Now, we analyze the behavior of the system when the disturbance is applied to the load. At $t=30 \mathrm{~ms}$, the resistance $R$ changes from $200 \Omega$ to $350 \Omega$ and it is forced to change again at $t=60 \mathrm{~ms}$ to $80 \Omega$. The results of this numerical experiment are depicted in Figure 8. As before, the system is able to handle the disturbances and it reaches the desired value of the output voltage in $t \approx 10 \mathrm{~ms}$, depending on the size of the disturbance. A close look to the function $s(x(t))$ in the upper inset of Figure $8 \mathrm{~B}$ reveals the effect of the secondary transitions within the same position of the MOSFET in the shape of $s(x(t))$. Here, one can see the appearance of a further linear segment, which, nevertheless, does not substantially affect the three-segment piece-wise linear approximation used here. This can be testified by the small errors that are reported in the main panel shown in Figure 8B.
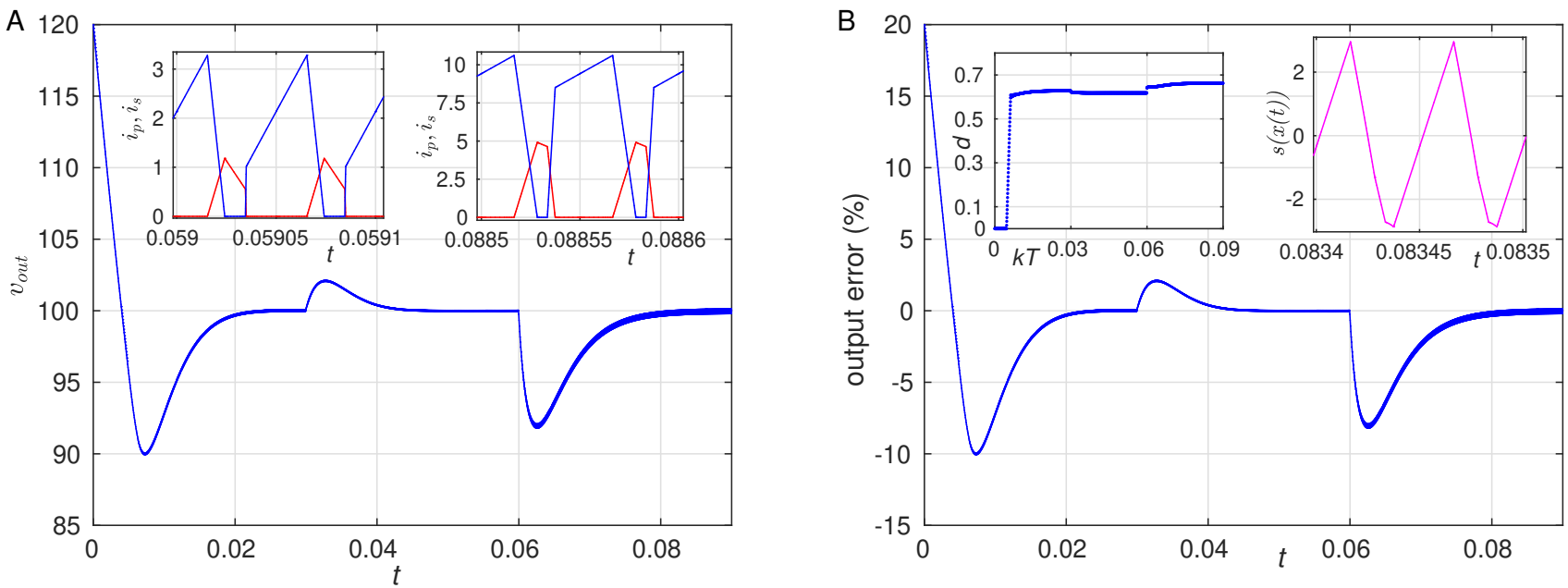

Figure 8. (A). Output voltage as a function of time with a disturbance in the resistance presented at $t=0.03 \mathrm{~s}$ and $t=0.06$ s. Insets: primary (secondary) currents depicted in blue (red) before and after the change in $v_{\text {out }}$. (B). Output error as a function of time for the evolution of $v_{\text {out }}$ in panel (A). Lower inset: dynamics of the duty cycle. Upper inset: function $s(x(t))$ defined in Equation (2). In this figure, the parameters have been chosen as in Table 2, with load variation from $R=200 \Omega$ to $R=350 \Omega$ to $R=80 \Omega$. 
Finally, the last disturbance involves, simultaneously, changes in the reference value, load resistances as well as in the input voltage. Firstly, at $t=30 \mathrm{~ms}, v_{\text {ref }}$ changes to $120 \mathrm{~V}$, $R$ changes to $350 \Omega$ and $v_{\text {in }}=8 \mathrm{~V}$. Secondly, at $t=60 \mathrm{~ms}$, the reference voltage changes to $v_{\text {ref }}=80 \mathrm{~V}$, the input voltage changes to $v_{\text {in }}=25 \mathrm{~V}$, and the load changes to $R=80 \Omega$. Figure 9 shows the results of this experiment. In this figure, it is possible to see that, despite the sudden transitions in a large proportion of the system's parameters, it evolves satisfactorily to the desired orbit. For the sake of visualization, we have included the whole time series of the function $s(x(t))$ in order to show that it has indeed a zero-average behavior in steady state, as hypothesized by the ZAS strategy.
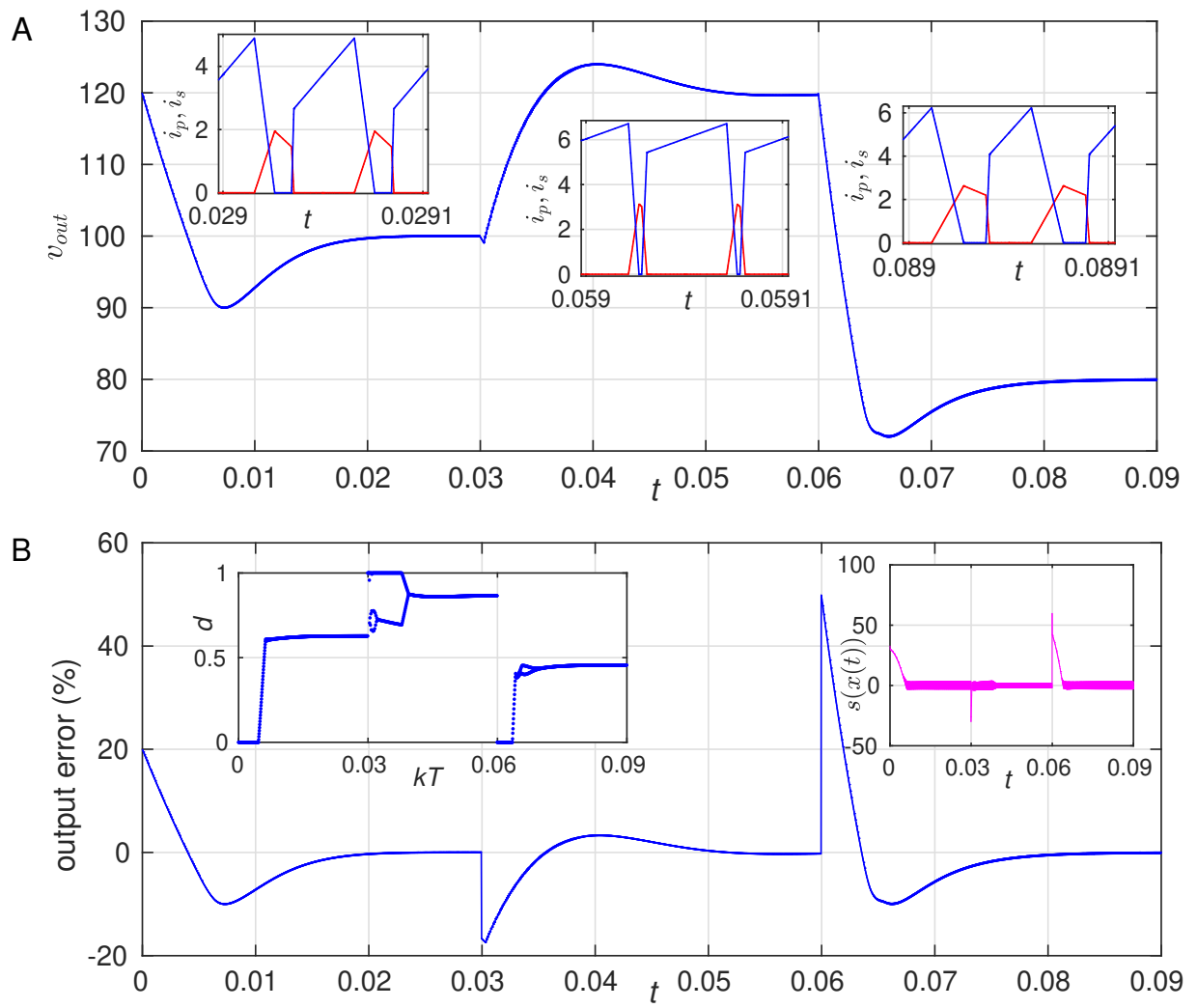

Figure 9. (A). Main panel: output voltage as function of time with disturbances performed at the reference voltage, the input voltage and the load simultaneously. The disturbances are applied at $t=30 \mathrm{~ms}$ and $t=60 \mathrm{~ms}$ according to the description in the main text. Insets: Primary (secondary) currents depicted in blue (red) during the steady operation after the transitions. (B). Main panel: output error for the disturbances in (A). Insets: duty cycle evolution (left) and time series over the whole interval of the function $s(x(t))$ (right). The parameters, as in Table 2.

From the numerical experiments of this section, we can assert that the basin of attraction of the period- 2 solution is small when compared with the basin of the period- 1 orbit. This can be inferred, because, according to the bifurcation diagrams described in Section 4, the period-2 solution should be present in the different disturbances applied here; however, the solution always evolved towards the desired period- 1 solution.

\section{Conclusions and Future Work}

In this work, we have successfully applied the Zero Average Surface (ZAS) controller for output regulation in a boost-flyback converter. The strategy consisted of the definition of a state-dependent function - $s(x(t))$-with the most information of the system's state. The ZAD control technique (precursor of the ZAS strategy presented here) was originally used to force a function that depends on the error and its derivative to have a zero-average in a PWM cycle $[20,21]$. Here, we have shown that adding an integral control action and 
imposing in every cycle a zero-average of a strategically chosen combination of the states, renders the system quite robust over a wide interval of input voltage, reference voltage, and load. Moreover, the original ZAD has been used to control bilinear systems [21-24,32], where the system is only represented by two topologies. Nevertheless, when the converter has a more complex topology, as in the case of the boost-flyback converter, the computation of the duty cycle gets more difficult. In contrast, with the ZAS technique, we have been able to obtain a simple algebraic expression of the duty cycle while using few approximations of the converter's dynamics and the state of the system at the beginning of the cycle. The duty cycle expression can be easily implemented on an embedded hardware system in order to generate a PWM signal, where the state variables are only sensed at the beginning of the switching period.

One of the main advantages of the ZAS controller is that it is designed to operate at a fixed frequency (the inverse of the sampling period $T$ ). Other controllers that make use of a variable frequency introduce undesired frequency-dependent harmonics to the signal. Eliminating such harmonics require the addition of different filters that add to the complexity of the overall system. Moreover, variable frequency techniques require semiconductor devices that fit within the ranges of commutation frequencies in order to guarantee a correct operation. For these reasons, the ZAS controller is a desirable strategy when the harmonics of the output signal are undesired.

Despite the fact that the function $s(x(t))$ provides information of all five states of the system, it only relies on the tuning of three parameters (two for the proportional terms and one for the integral term). These can be further reduced to two parameters by means of a properly selected normalization of the parameters. Nonetheless, we have chosen, during the development of this work, to write all of the parameters down explicitly to keep track of the individual contributions of the terms in the function $s(x(t))$.

Even though the boost-flyback exhibits three different stable period-1 orbits, we have seen that the orbit 5436 was consistently the most robust across the experiments. Additionally, we reported that the stability of the orbit was lost by means of a period doubling and a subsequent saturation of the duty cycle at high values of the converter's gain. In our numerical experiments with varying $v_{i n}$ and $v_{r e f}$, the value of the gain at which the bifurcation occurred was quite similar, namely $\approx 14$. This seems to suggest that the boost-flyback converter controlled via the ZAS strategy is adequate for intermediate output voltage gains, and different controllers should be used for higher gains.

We showed that the robustness of the 5436 orbit is related with the larger region of attraction in the state space (basin of attraction) when compared with other coexisting attractors. This is consistent with previously reported results (see $[12,13,17,19])$. The calculation of the precise size of the basin of attraction is not an easy task, and it usually involves the application of the so-called Lyapunov direct methods, which are hard to apply, especially in non-smooth systems. In the case in which a single attractor is found, it is also non-trivial to determine whether this attractor is either globally or locally stable. Recent techniques that are based on contraction theory [33] allow for assessing global stability, even in non-smooth systems [34,35], including power converters [36]. Similar approaches could be applied to the boost-flyback converter in future research.

In the bifurcation diagrams varying the load, we suggested the presence of a chaotic attractor in a small window of the diagram. This result, together with other preliminary experiments-not shown here-performed at very low values of the parameters bring to light the need of a more complete characterization of the system. Such characterization must be performed, not only in terms of Floquet multipliers, but also Lyapunov Exponents as a preliminary step before an experimental implementation. This is part of an ongoing work that presents some challenges itself: For instance, the (formal) calculation of the Floquet multipliers and Lyapunov exponents require the use of the saltation matrix formalism for the correction of the linearised evolution of the system (perturbation's dynamics), due to the discontinuities in the vector fields [28,29]. While this problem is relatively easy to solve in the cases in which the switching from one vector field to another is defined by a precise 
value of the state at the time of the discontinuity, we are here approximating the time of the discontinuity as a function of the states in the previous cycle. This calls for the need of a different approach to assess linear stability, for example, via the direct linearisation of the event driven map, which has been successfully used in applications involving pulse coupled oscillators [37,38].

In summary, the ZAS technique is ideal for applications that require: (i) intermediate gains, (ii) large operation region, and (iii) disturbance rejection. Examples of devices that required these specifications include hybrid electric vehicles [39,40], voltage balancing [41], photovoltaic panels [42], LED technologies [43], and correction of the power factor [44]. Finally, with the aim of contextualizing the contribution of this work, we present, in Table 3, a summary of the advantages and disadvantages of different controllers that have been applied to the boost-flyback, and how they compare with the ZAS technique that was used here.

Table 3. Advantages and disadvantages of different control techniques used for the boost-flyback converter in the literature.

\begin{tabular}{lll}
\hline $\begin{array}{l}\text { Control Techniques for the } \\
\text { Boost-Flyback }\end{array}$ & Advantages & Disadvantages \\
\hline $\begin{array}{l}\text { Current Control and Voltage } \\
\text { Control (small signal model) [45] }\end{array}$ & $\begin{array}{l}\text { Fixed Switching } \\
\text { Frequency, perturbation } \\
\text { rejected }\end{array}$ & $\begin{array}{l}\text { Only small scale } \\
\text { instabilities are } \\
\text { considered }\end{array}$ \\
\hline Sliding Control [46] & $\begin{array}{l}\text { Robustness, disturbance } \\
\text { rejection }\end{array}$ & $\begin{array}{l}\text { Unfixed switching } \\
\text { frequency }\end{array}$ \\
\hline Peak current-mode control [17] & $\begin{array}{l}\text { Fixed switching } \\
\text { Frequency, disturbance } \\
\text { rejection }\end{array}$ & $\begin{array}{l}\text { Slope compensation is } \\
\text { required and } \\
\text { operation zone is } \\
\text { bounded }\end{array}$ \\
\hline Hysteresis band control [19] & $\begin{array}{l}\text { Robustness, disturbance } \\
\text { rejection }\end{array}$ & $\begin{array}{l}\text { Unfixed switching } \\
\text { frequency }\end{array}$ \\
\hline & $\begin{array}{l}\text { Robustness, disturbance } \\
\text { rejection, simple } \\
\text { implementation } \\
\text { (straightforward duty } \\
\text { cycle expression), known } \\
\text { fixed frequency }\end{array}$ & $\begin{array}{l}\text { Operation zone is } \\
\text { bounded }\end{array}$ \\
ZAS control & & \\
\hline
\end{tabular}

Author Contributions: Conceptualization: J.-G.M. and F.A.; Formal analysis, J.-G.M., F.A. and D.A.-G.; Investigation, J.-G.M., F.A. and D.A.-G.; Methodology, J.-G.M.; Project administration, F.A.; Software, J.-G.M.; Visualization, D.A.-G.; Writing-original draft, F.A. and D.A.-G.; Writingreview and editing, F.A. and D.A.-G. All authors have read and agreed to the published version of the manuscript.

Funding: F.A. was supported by Universidad Nacional de Colombia, Manizales, Project 46277Vicerrectoría de Investigación. J.-G.M. received support by Instituto Tecnológico Metropolitano through Project ITM-P17224.

Conflicts of Interest: The authors declare no conflict of interest.

\section{References}

1. Gandhi, V.I.; Subramaniyaswamy, V.; Logesh, R. Topological review and analysis of DC-DC boost converters. J. Eng. Sci. Technol. 2017, 12, 1541-1567.

2. Guepfrih, M.F.; Waltrich, G.; Lazzarin, T.B. Quadratic-boost-double-flyback converter. IET Power Electron. 2019, $12,3166-3177$. [CrossRef]

3. Periyanayagam, M.; Kumar V, S.; Chokkalingam, B.; Padmanaban, S.; Mihet-Popa, L.; Adedayo, Y. A Modified High Voltage Gain Quasi-Impedance Source Coupled Inductor Multilevel Inverter for Photovoltaic Application. Energies 2020, 13, 874. [CrossRef] 
4. Chen, Z.; Zhou, Q.; Xu, J. Coupled-inductor boost integrated flyback converter with high-voltage gain and ripple-free input current. Power Electron. IET 2015, 8, 213-220. [CrossRef]

5. Liu, H.; Su, X.; Wang, J. High Step-up Coupled Inductor Inverters Based on qSBIs. Energies 2019, 12, 3032. [CrossRef]

6. Li, W.; Xiao, J.; Wu, J.; Liu, J.; He, X. Application Summarization of Coupled Inductors in DC/DC Converters. In Proceedings of the 2009 Twenty-Fourth Annual IEEE Applied Power Electronics Conference and Exposition, Washington, DC, USA, 15-19 February 2009; pp. 1487-1491.

7. Zhao, Q.; Lee, F. High performance coupled-inductor DC-DC converters. In Proceedings of the Applied Power Electronics Conference and Exposition, 2003. APEC '03. Eighteenth Annual IEEE, Miami Beach, FL, USA, 9-13 February 2003; Volume 1, pp. 109-113.

8. Tseng, K.; Liang, T. Novel high-efficiency step-up converter. Electr. Power Appl. IEE Proc.-Appl. 2004, 151, 182-190. [CrossRef]

9. Liang, T.; Tseng, K. Analysis of integrated boost-flyback step-up converter. Electr. Power Appl. IEE Proc.-Appl. 2005, 152, 217-225. [CrossRef]

10. Carrero Candelas, N.A. Modelado, Simulación y Control de un Convertidor Boost Acoplado Magnéticamente. Ph.D. Thesis, Universidad Politécnica de Catalunya, Barcelona, Spain, 2014. Available online: https://upcommons.upc.edu/handle/2117/953 92? show =full (accessed on 20 September 2020).

11. Carrero, N.; Batlle, C.; Fossas, E. Averaged dynamics of a coupled-inductor boost converter under sliding mode control using a piecewise linear complementarity model. IMA J. Appl. Math. 2016, 81, 688-698. [CrossRef]

12. Muñoz, J.G.; Gallo, G.; Osorio, G.; Angulo, F. Performance Analysis of a Peak-Current Mode Control with Compensation Ramp for a Boost-Flyback Power Converter. J. Control Sci. Eng. 2016, 2016, 7354791. [CrossRef]

13. Muñoz Cataño, J.G. Análisis Dinámico y Control de un Convertidor Boost-Flyback. Ph.D. Thesis, Universidad Nacional de Colombia-Sede Manizales, Caldas, Colombia, 2017. Available online: https://repositorio.unal.edu.co/handle/unal/63019 (accessed on 20 September 2020).

14. Bernardo, M.; Budd, C.; Champneys, A.; Kowalczyk, P. Piecewise-smooth Dynamical Systems, Theory and Applications; Springer: Berlin/Heidelberg, Germany, 2008.

15. Leine, R.; van Campen, D.; van de Vrande, B. Bifurcations in Nonlinear Discontinuous Systems. Nonlinear Dyn. 2000, 23, 105-164 [CrossRef]

16. Giaouris, D.; Banerjee, S.; Zahawi, B.; Pickert, V. Stability Analysis of the Continuous-Conduction-Mode Buck Converter Via Filippov's Method. IEEE Trans. Circuits Syst. I Regul. Pap. 2008, 55, 1084-1096. [CrossRef]

17. Muñoz, J.G.; Gallo, G.; Angulo, F.; Osorio, G. Slope Compensation Design for a Peak Current-Mode Controlled Boost-Flyback Converter. Energies 2018, 11, 3000. [CrossRef]

18. Muñoz, J.G.; Pérez, A.; Angulo, F. Enhancing the Stability of the Switched Systems Using the Saltation Matrix. Int. J. Struct. Stab. Dyn. 2019, 19, 1941004. [CrossRef]

19. Muñoz, J.G.; Angulo, F.; Angulo-Garcia, D. Designing a hysteresis band in a boost flyback converter. Mech. Syst. Signal Process. 2021, 147, 107080. [CrossRef]

20. Fossas, E.; Griñó, R.; Biel, D. Quasi-Sliding control based on pulse width modulation, zero averaged dynamics and the L2 norm. In Advances In Variable Structure Systems: Analysis, Integration and Applications; World Scientific: Singapore, 2000; pp. 335-344.

21. Angulo, F. Análisis de la Dinámica de Convertidores Electrónicos de Potencia Usando PWM Basado en Promediado Cero de la Dinámica del Error (ZAD). Ph.D. Thesis, Universidad Politécnica de Cataluña, Barcelona, Spain, 2004. Available online: www.tdx.cat/handle/10803/5941 (accessed on 20 September 2020).

22. Angulo, F.; Ocampo, C.; Olivar, G.; Ramos, R. Nonlinear and nonsmooth dynamics in a DC-DC Buck converter: Two experimental set-ups. Nonlinear Dyn. 2006, 46, 239-257. [CrossRef]

23. Hoyos, F.; Angulo, F.; Taborda, J.; Olivar, G. Implementation of a new digital control technique for DC-DC and DC-AC power converters. Dyna 2010, 77, 189-199.

24. Angulo, F.; Olivar, G.; Taborda, J.; Hoyos, F. Nonsmooth dynamics and FPIC chaos control in a DC-DC ZAD-strategy power converter. In Proceedings of the Sixth Euromech Nonlinear Dynamics Conference (Enoc 2008), Citeseer, Saint Petersburg, Russia, 30 June-4 July 2008; Volume 55, pp. 2392-2401.

25. Mohan, N. First Course on Power Electronics and Drives; Mnpere: Minneapolis, MN, USA, 2003.

26. Angulo, F.; Fossas, E.; Olivar, G. Transition from periodicity to chaos in a PWM-controlled buck converter with ZAD strategy. Int. J. Bifurc. Chaos 2005, 15, 3245-3264. [CrossRef]

27. Erickson, R.W.; Maksimovic, D. Fundamentals of Power Electronics; Springer Science \& Business Media: Berlin/Heidelberg, Germany, 2007.

28. El Aroudi, A.; Giaouris, D.; Iu, H.H.C.; Hiskens, I.A. A review on stability analysis methods for switching mode power converters. IEEE J. Emerg. Sel. Top. Circuits Syst. 2015, 5, 302-315. [CrossRef]

29. Leine, R.I.; Nijmeijer, H. Dynamics and Bifurcations of Non-Smooth Mechanical Systems; Springer: Berlin/Heidelberg, Germany, 2004.

30. Guckenheimer, J.; Holmes, P. Nonlinear Oscillations, Dynamical systems and Bifurcations of Vector Fields; Springer: Berlin/Heidelberg, Germany, 1983.

31. Gallo Hernández, G.L. Simulación Automática de Convertidores Electrónicos de Potencia DC-DC. Ph.D. Thesis, Universidad Nacional de Colombia-Sede Manizales, Caldas, Colombia, 2017. Available online: https://repositorio.unal.edu.co/handle/unal/ 62965 (accessed on 20 September 2020). 
32. Muñoz, J.; Osorio, G.; Angulo, F. Boost converter control with ZAD for power factor correction based on FPGA. In Proceedings of the 2013 Workshop on Power Electronics and Power Quality Applications (PEPQA), Bogota, Colombia, 6-7 July 2013; pp. 1-5. [CrossRef]

33. Lohmiller, W.; Slotine, J.J.E. On contraction analysis for non-linear systems. Automatica 1998, 34, 683-696. [CrossRef]

34. Fiore, D.; Hogan, S.J.; Di Bernardo, M. Contraction analysis of switched systems via regularization. Automatica 2016, 73, 279-288. [CrossRef]

35. Di Bernardo, M.; Fiore, D. Switching control for incremental stabilization of nonlinear systems via contraction theory. In Proceedings of the 2016 European Control Conference (ECC), Aalborg, Denmark, 29 June-1 July 2016; pp. 2054-2059.

36. Angulo-Garcia, D.; Angulo, F.; Osorio, G.; Olivar, G. Control of a dc-dc buck converter through contraction techniques. Energies 2018, 11, 3086. [CrossRef]

37. Angulo-Garcia, D.; Torcini, A. Stochastic mean-field formulation of the dynamics of diluted neural networks. Phys. Rev. E 2015, 91, 022928. [CrossRef] [PubMed]

38. Zillmer, R.; Livi, R.; Politi, A.; Torcini, A. Stability of the splay state in pulse-coupled networks. Phys. Rev. E 2007, 76, 046102. [CrossRef]

39. Lai, C.M.; Yang, M.J. A High-Gain Three-Port Power Converter with Fuel Cell, Battery Sources and Stacked Output for Hybrid Electric Vehicles and DC-Microgrids. Energies 2016, 9, 180. [CrossRef]

40. Tseng, K.C.; Lin, J.T.; Cheng, C.A. An Integrated Derived Boost-Flyback Converter for fuel cell hybrid electric vehicles. In Proceedings of the 2013 1st International Future Energy Electronics Conference (IFEEC), Tainan, Taiwan, 3-6 November 2013; pp. $283-287$.

41. Park, J.H.; Kim, K.T. Multi-output differential power processing system using boost-flyback converter for voltage balancing. In Proceedings of the 2017 International Conference on Recent Advances in Signal Processing, Telecommunications Computing (SigTelCom), Da Nang, Vietnam, 9-11 January 2017; pp. 139-142. [CrossRef]

42. Chen, S.M.; Wang, C.Y.; Liang, T.J. A novel sinusoidal boost-flyback CCM/DCM DC-DC converter. In Proceedings of the 2014 IEEE Applied Power Electronics Conference and Exposition-APEC, Fort Worth, TX, USA, 16-20 March 2014; pp. 3512-3516.

43. Lee, S.W.; Do, H.L. A Single-Switch AC-DC LED Driver Based on a Boost-Flyback PFC Converter with Lossless Snubber. IEEE Trans. Power Electron. 2017, 32, 1375-1384. [CrossRef]

44. Divya, K.M.; Parackal, R. High power factor integrated buck-boost flyback converter driving multiple outputs. In Proceedings of the 2015 Online International Conference on Green Engineering and Technologies (IC-GET), Coimbatore, India, 27 November 2015; pp. 1-5. [CrossRef]

45. Brockveld, S.L.; Waltrich, G. Boost-flyback converter with interleaved input current and output voltage series connection. IET Power Electron. 2018, 11, 1463-1471. [CrossRef]

46. Carrero, N.; Batlle, C.; Fossas, E. Experimental evaluation of a cascade sliding mode-PI controller for a coupled-inductor Boost converter. In Proceedings of the 2014 13th International Workshop on Variable Structure Systems (VSS), Nantes, France, 29 June2 July 2014; pp. 1-6. [CrossRef] 